\title{
Autoantibodies to Nuclear Envelope Antigens in Chronic Fatigue Syndrome
}

\author{
K. Konstantinov, A. von Mikecz, D. Buchwald, ${ }^{\star}$ J. Jones, ${ }^{\ddagger}$ L. Gerace, and E.M. Tan \\ Autoimmune Disease Center and Department of Cell Biology, The Scripps Research Institute, La Jolla, California 92037; *University of \\ Washington School of Medicine, Seattle, Washington 98104; and ${ }^{\ddagger}$ National Jewish Center, Denver, Colorado 80206
}

\begin{abstract}
We have identified and partially characterized the autoantibodies in sera of 60 patients with chronic fatigue syndrome. Approximately $52 \%$ of the sera were found to react with nuclear envelope antigens. The combination of nuclear rim staining observed in immunofluorescence microscopy and immunoblot analysis of highly purified nuclear envelope proteins provided initial characterization of these autoantibodies. Further characterization showed that some sera immunoprecipitated the in vitro transcription and translation product of a human cDNA clone encoding the nuclear envelope protein lamin B1. The autoantibodies were of the IgG isotype. The occurrence of autoantibodies to a conserved intracellular protein like lamin B1 provides new laboratory evidence for an autoimmune component in chronic fatigue syndrome. (J. Clin. Invest. 1996. 98:1888-1896.) Key words: autoantibodies • chronic fatigue syndrome - nuclear lamins - nuclear envelope antigens
\end{abstract}

\section{Introduction}

Chronic fatigue syndrome $(\mathrm{CFS})^{1}$ is a clinically defined condition, consisting of profound fatigue associated with a variety of nonspecific and constitutional symptoms (1-3). CFS affects up to 267 per 100,000 people (4) and can occur sporadically or in clusters. Diagnosis of CFS can be made only after alternative medical and psychiatric causes have been excluded. So far, no pathognomonic signs or diagnostic tests have proven to be useful for recognizing the illness or for studies to elucidate its etiologic or pathogenic mechanisms. Routine tests are usually normal, and the recommended evaluation includes physical examination, CBC and differential, ESR, complete blood chemistry, urinalysis, chest roentgenograms, personal and family psychiatric history, and determination of thyroid-stimulating hormone (3) to exclude another condition that might explain abnormalities in any of these tests.

Address correspondence to Eng M. Tan, M.D., The Scripps Research Institute, La Jolla, CA 92037. Phone: 619-784-8686; FAX: 619-7842131.

Received for publication 12 April 1996 and accepted in revised form 29 July 1996.

1. Abbreviations used in this paper: ANAs, antinuclear antibodies; CFS, chronic fatigue syndrome; IIF, indirect immunofluorescence; LAP, lamina-associated polypeptide; NE, nuclear envelope; NPC, nuclear pore complex; PO, phosphoprotein component; SWNEs, saltwashed nuclear envelopes.

J. Clin. Invest.

(C) The American Society for Clinical Investigation, Inc. 0021-9738/96/10/1888/09 \$2.00

Volume 98, Number 8, October 1996, 1888-1896
Some manifestations of CFS suggest that it may be associated with immunologic dysfunction. These include abnormalities or alterations in T cell function (5), natural killer cell cytotoxicity (6), cytokine production (7), mitogen stimulation (6), and lymphocyte phenotypes (8). The presence of circulating immune complexes and antinuclear antibodies (ANAs) in as high as $30 \%$ of patients with CFS has also been reported (9). While each of these laboratory tests lacks sufficient sensitivity and specificity to be diagnostic, the immunologic abnormalities described are in accordance with a growing body of evidence suggesting chronic, low-level activation of the immune system in CFS. Some investigators also suggest that immune dysfunction leading to chronic infection might be an etiologic factor $(10,11)$.The association of CFS and a disorder resembling Sjogren's syndrome with positive Schirmer's test, ANA, and abnormal salivary gland biopsy, but absence of SS-A/Ro antibodies, has been described (12).

We have found that $\sim 52 \%$ of patients with CFS develop autoantibodies to components of the nuclear envelope (NE), mainly nuclear lamins. Our findings suggest that in addition to other disturbances of the immune system, humoral autoimmunity against polypeptides of the NE is a prominent immune derangement in CFS.

\section{Methods}

CFS patients. CFS patients were recruited from a university-based fatigue clinic in the Pacific Northwest which accepts both self- or physician referrals. Patients underwent an extensive medical and psychological intake evaluation as recommended by the Centers for Disease Control and Prevention (CDC), National Institutes of Health, and the National Institute of Mental Health $(13,14)$. This evaluation consisted of a standardized physical examination which included palpation of tender points, a questionnaire specific to CFS symptoms also containing items on past and current medical problems, routine blood tests, and a structured psychiatric interview which yielded DSM-III-R diagnoses. Each subject was also interviewed by a physician assistant and by one of us (D. Buchwald). The laboratory battery included a complete blood count, erythrocyte sedimentation rate, chemistry panel, liver and thyroid function tests, and ANAs. In addition, patients underwent an unanesthetized Schirmer's test. Other studies were ordered as clinically indicated.

Psychiatric diagnoses were determined using the National Institutes of Mental Health Diagnostic Interview Schedule Version III-A (13). The Diagnostic Interview Schedule is a widely used, highly structured interview which assigns current and lifetime diagnoses based on DSM-III-R criteria (14). An abridged version with sections on major depression, alcohol abuse, agoraphobia and somatization, generalized anxiety, dysthymic, and panic disorders was administered by a psychometrist trained in its use.

Patients were classified as having CFS using the 1988 criteria (1) and subsequent modifications (2) which explicitly do not exclude most psychiatric disorders. The presence of affective, anxiety, and somatization disorders did not exclude patients from meeting the CDC criteria for CFS. However, as specified by the criteria, patients who met the CDC criteria had no evidence of a medical condition or previously documented schizophrenia, bipolar depression, or personality 
disorders which could explain their fatigue. The 1988 case definition requires the presence of persistent or recurrent, disabling $(50 \%$ reduction of pre-morbid activity levels) fatigue lasting at least 6 mo which is not explained by exertion or circumstances. In addition, at least 8 of the following 11 symptoms must be present: 1 , mild fever between 99.4 and $101.4^{\circ} \mathrm{C}$ or chills; 2, sore throat; 3, painful cervical or axillary lymph nodes; 4 , unexplained, diffuse, muscle weakness; 5 , muscle pain or discomfort; 6 , new or different regular headaches; 7 , noninflammatory, multi-joint aches; 8 , postexertional fatigue/malaise; 9, neurocognitive difficulties (e.g., difficulty concentrating); 10, problems falling or staying asleep or sleeping too much; 11, acute onset. Of note, the newly formulated 1994 international CDC case definition (3) has similar psychiatric and medical exclusionary criteria and incorporates the major components, including symptoms of the original criteria (1). The new definitions were not used because they had not been published at the time this study was commenced and because they require the collection of some data (e.g., patient height) that were not routinely obtained by our group (or others) at the time.

Control subjects. Controls consisted of two groups (Table I). The first control group (I) was a community-based sample of individuals not seeking medical care and recruited by newspaper advertisements for participation in a research study in Denver, CO. This group, comprising 30 individuals ( 10 with current major depression, 10 with allergies, and 10 in good health), was selected since affective and allergic conditions are prominent features of CFS. All subjects in control group I underwent a standardized evaluation including a structured psychiatric assessment, physical examination, skin testing, and laboratory studies. No subject had evidence of a primary active medical disorder (except allergies). Subjects with allergies and healthy controls denied fatigue and did not meet criteria for any current or lifetime psychiatric illness. No subject in this control group met the case definition for CFS.

The Seattle (CFS patients) and Denver (control group I patients) patients came from two centers which are part of an NIH-funded CFS Cooperative Research Group. One of the objectives of the Clinical Cores of these centers was to develop banks of sera and clinical data as a source of patients for other investigations. Although the CFS sera and the control group I sera came from different geographical locations, they were collected following guidelines established by the CFS Study Group and should be comparable from one center to another.

The second control group (II) consisted of 41 blood donors at the General Clinical Research Center at the Scripps Clinic and The Scripps Research Institute. These blood donors came from a cohort of healthy individuals who had participated in many studies as normal controls. These donors were employees who were screened at least annually for blood counts and for HBV, HCV, and HIV serology. They were known to be working on a regular basis and would there- fore not meet the CFS case definition, because they would not meet the sine qua non criterion of persisting or relapsing fatigue for $\geq 6 \mathrm{mo}$.

Informed consent was obtained from all subjects and approval was obtained from the appropriate Institutional Review Board. Serum was the only patient material used in the laboratory analysis and since antibodies are stable during storage in the frozen condition, transportation of sera from Seattle and Denver was always in the frozen state.

Reference antibodies. Mouse monoclonal antibody (RL12, IgM) to lamins A/C (15), Armenian hamster monoclonal antibody (RL29, $\mathrm{IgG})$ to lamina-associated polypeptide 2 (LAP $2 ; 16$ ), mouse monoclonal antibody (RL1, IgM) to a group of nuclear pore complex (NPC) glycoproteins (17), mouse monoclonal antibody (V-7505; Sigma Chemical Co., St. Louis, MO; IgG) to vimentin, rabbit polyclonal antibody to p80 coilin (18), and a previously characterized anti-lamin B serum from a patient with systemic lupus erythematosus (19) were used as reference antibodies. The NE specific monoclonal antibodies (RL12, RL29, and RL1) were prepared against proteins of a pore complex-lamina fraction from rat liver NEs as described (15). Antilamin and anti-NPC monoclonal antibodies were prepared by immunization of mice, while anti-LAP 2 antibodies were produced by immunization of an Armenian hamster.

Indirect immunofluorescence (IIF) microscopy. Human HeLa and HEp-2 cells were obtained from the American Type Culture Collection (Rockville, MD) and grown in DMEM containing 10\% fetal bovine serum at $37^{\circ} \mathrm{C}$ in a $5 \% \mathrm{CO}_{2}$ atmosphere. IIF microscopy was performed on HeLa or HEp-2 cells grown on coverslips or commercially available prefixed HEp-2 cell substrates (Bion, Park Ridge, IL). Coverslips and commercial slides were incubated with the human sera starting at serum dilution of 1:50 and followed by titration to end point in $4 \%(\mathrm{wt} / \mathrm{vol})$ nonfat dry milk in PBS. Incubation was carried out at $25^{\circ} \mathrm{C}$ for $1 \mathrm{~h}$. Double IIF was performed by mixing a human serum with one of the following: monoclonal antivimentin (mouse), anti-LAP 2 (Armenian hamster), or anti-NPC (mouse) antibodies at $10 \mu \mathrm{g} / \mathrm{ml}$ or rabbit anti-p80 coilin antibodies diluted 1:100. After washing three times for $10 \mathrm{~min}$ in PBS, the substrates were incubated with a 1:100 dilution of the appropriate secondary antibodies (FITC or rhodamine-conjugated goat anti-human, anti-mouse, or anti-rabbit Fab-specific IgGs; Caltag Laboratories, San Francisco, CA) for $1 \mathrm{~h}$ at $25^{\circ} \mathrm{C}$, washed with PBS, and mounted in Vectashield (Vector Laboratories, Inc., Burlingame, CA). Antibody class was determined by using anti-IgG, anti-IgM, and anti-IgA detecting reagents. DNA was counterstained by including $1 \mu \mathrm{g} / \mathrm{ml}$ 4 $^{\prime} 6$-diamidino-2-phenylindole (DAPI; Sigma Chemical Co.) in the last PBS wash. Slides were examined under an Olympus BH-2 fluorescence microscope and photographed with Kodak Ektachrome 400 film. FITC and rhodamine fluorochromes were merged with a triple filter to determine presence or absence of antigen colocalization.

Table I. Demographic Data on Subjects

\begin{tabular}{|c|c|c|c|c|c|}
\hline \multirow[b]{2}{*}{ Feature } & \multirow{2}{*}{$\begin{array}{c}\text { CFS } \\
\begin{array}{c}\text { CFS } \\
(n=60)\end{array}\end{array}$} & \multicolumn{2}{|c|}{ Control group I } & \multicolumn{2}{|c|}{ Control group II } \\
\hline & & $\begin{array}{l}\text { Allergy } \\
(n=10)\end{array}$ & $\begin{array}{c}\text { MD } \\
(n=10)\end{array}$ & $\begin{array}{c}\mathrm{HC} \\
(n=10)\end{array}$ & $\begin{array}{c}\text { BD } \\
(n=41)\end{array}$ \\
\hline Age (yr) & 38.0 & 41.3 & 42.7 & 45.0 & 37.1 \\
\hline Gender ( $\%$ female $)$ & $51(85)$ & $9(90)$ & $9(90)$ & $10(100)$ & $23(56)$ \\
\hline Race/ethnicity (\% Caucasian) & $54(90)$ & $8(80)$ & $10(100)$ & $9(90)$ & $32(78)$ \\
\hline Illness duration, mean years & 4.0 & ND & ND & 0 & 0 \\
\hline Concurrent psychiatric disorder (\%) & $30(50)$ & 0 & 100 & 0 & ND \\
\hline Concurrent FM (\%) & $30(50)$ & 0 & 0 & 0 & ND \\
\hline Positive Schirmer's test (\%) & $17(28)$ & ND & ND & ND & ND \\
\hline Concurrent allergies (\%) & $33(58)$ & 100 & $9(90)$ & 0 & ND \\
\hline
\end{tabular}

$M D$, major depression; $H C$, healthy controls; $B D$, blood donors; $N D$, no data. 
In the immunofluorescence assay, control negative sera as well as control positive sera were always included. Also, each serum which was positive was titrated in doubling dilutions to determine the end point titer of the antibody. This was an added factor which gave a semiquantitative estimation of the antibody content. The immunofluorescence readings were made initially by two of the authors (K. Konstantinov and A. von Mikecz) and were further evaluated in equivocal cases by a technician with $20 \mathrm{yr}$ of experience in reading immunofluorescent ANAs and by a senior author (E.M. Tan).

Preparation of extracts. NEs were isolated from rat liver as described previously (20). In brief, this procedure consisted of fractionation of liver homogenate from Sprague Dawley rats to obtain highly purified nuclei. Crude NEs were obtained by successive DNase $(1 \mu \mathrm{g} / \mathrm{ml})$ and RNase $(1 \mu \mathrm{g} / \mathrm{ml})$ digestions. These preparation of NEs were depleted of weakly adherent components and contaminants by suspending $0.5-2 \mathrm{mg}$ in each $\mathrm{ml}$ of a high salt buffer containing $10 \mathrm{mM}$ Hepes, pH 7.4, $500 \mathrm{mM} \mathrm{NaCl}, 0.1 \mathrm{mM} \mathrm{MgCl}_{2}, 1 \mathrm{mM}$ dithiothreitol, and protease inhibitors $(1 \mathrm{mM}$ phenylmethylsulfonyl fluoride and $1 \mu \mathrm{g} / \mathrm{ml}$ aprotinin, pepstatin, and leupeptin). The suspension was incubated for $5 \mathrm{~min}$ at $4^{\circ} \mathrm{C}$ and spun for $20 \mathrm{~min}$ at 5,000 rpm in a JS 5.2 rotor (Beckman Instruments Inc., Palo Alto, CA) yielding a supernatant and pellet. The latter was designated "salt-washed NEs" (SWNEs). All fractionation procedures were conducted at $0-4^{\circ} \mathrm{C}$.

MOLT-4 cells were obtained from the American Type Culture Collection and grown in DMEM containing $10 \%$ fetal bovine serum. The MOLT- 4 whole cell extract was prepared as described previously (21). Briefly, cells grown in culture dishes were scraped into ice-cold PBS. After centrifugation, cell pellets were lysed in Laemmli's sample buffer (22) and sheared by passage through a fine needle. Samples were boiled for $5 \mathrm{~min}$ and cellular debris was removed by centrifugation at $15,000 \mathrm{~g}$.

Gel electrophoresis and immunoblotting. Samples were prepared for SDS-PAGE according to Laemmli (22). SWNEs were directly solubilized in SDS sample buffer. Samples containing 25-30 $\mu$ g protein per lane were separated under reducing conditions by SDS-PAGE on either 7.5 or $12.5 \%$ gels. Proteins were electrophoretically transferred to nitrocellulose (Schleicher \& Schuell Inc., Keene, NH) at $200 \mathrm{~mA}$ (60 $\mathrm{min}$ ) with a semidry blotting apparatus, containing transfer buffer (20\% methanol, $25 \mathrm{mM}$ Tris, $192 \mathrm{mM}$ glycine). Transferred proteins were visualized by staining the nitrocellulose sheets in $0.5 \%$ Ponceau $\mathrm{S}$, $3 \%$ trichloroacetic acid, followed by destaining in $\mathrm{H}_{2} \mathrm{O}$. Nitrocellulose strips were then blocked for $1 \mathrm{~h}$ at room temperature with $5 \%$ (wt/vol) nonfat milk in PBS containing $0.05 \%$ Tween-20 (PBST), and given sequential 1-h incubations at room temperature with patient sera, followed by peroxidase-conjugated goat anti-human IgG (Cappel Laboratories, Cochranville, PA) in blocking buffer. Primary antibodies were used in 1:100 dilution and secondary antibodies in 1:2,000 dilution. After each incubation, blots were given three 10-min washes in PBST to remove any unbound antibody. Bound antibody was detected by enhanced chemiluminescence (Amersham Corp., Arlington Heights, IL) according to the manufacturer's instructions. To obtain autoradiograms, immunoblots were first exposed to ECL reagent for $1 \mathrm{~min}$ immediately followed by a 3-min film exposure and development. Apparent molecular masses of proteins were determined from their mobilities when compared with prestained molecular mass protein standards (GIBCO BRL, Gaithersburg, MD).

Numerous experiments were carried out to optimize the immunoblotting conditions and to establish the specificity of data observed. We used sera from healthy subjects and CFS sera with weak and strong anti-NE reactivity to analyze how changes in the incubation time, composition of blocking buffer, concentrations of the primary antibodies, and length of autoradiographic film exposure affected the detection of specific immunoreactivities. We found that the use of $3 \%$ BSA/PBST as blocking buffer generated strong nonspecific diffuse background. Increased incubation time (overnight at $4^{\circ} \mathrm{C}$ ) with the primary antibodies (23) resulted in nonspecific extra bands in both the negative and positive control sera. Titration of the CFS sera showed optimal results at 1:100 dilution. Autoradiographic films were exposed to blots optimally for up to $3 \mathrm{~min}$, as longer exposures resulted in high background. In every experiment, negative and positive controls were included in order to ensure that each assay was consistent by reference to the performance of the controls.

Affinity purification of autoantibody fraction from nitrocellulose paper. Antibodies against the most common reactivity (68-kD region of SDS gels of SWNEs) were affinity purified from nitrocellulosebound antigen as described previously (24). Bound antibodies were eluted with $50 \mathrm{mM}$ glycine, $150 \mathrm{mM}$ sodium chloride ( $\mathrm{pH} 2.3$ ) and immediately neutralized with $0.5 \mathrm{M}$ sodium phosphate (pH 7.7). Eluted antibodies were concentrated with Centricon 100 microconcentrator (Amicon, Inc., Beverly, MA) and used without dilution for immunofluorescence microscopy or diluted 1:2 for immunoblotting.

Immunoprecipitation using in vitro translated human lamin B1 as antigen. $\left[{ }^{35} \mathrm{~S}\right]$ Methionine-labeled human lamin $\mathrm{B} 1$ was produced by in vitro transcription and translation from a cDNA clone previously isolated in this laboratory (19). RNA transcripts were synthesized in the presence of T3 polymerase using the TNT kit (Promega, Madison, WI) according to the manufacturer's instructions. Briefly, in vitro translation products were obtained by incubation of $2 \mu \mathrm{g}$ of lamin B1 cDNA $(1 \mu \mathrm{g} / \mu \mathrm{l})$ in $50 \mu \mathrm{l}$ of rabbit reticulocyte lysate (Promega) containing an amino acid mixture without methionine, but with added $\left.{ }^{35} \mathrm{~S}\right]$ methionine (ICN Radiochemicals, Irvine, CA) and RNasin in a $100 \mu \mathrm{l}$ reaction volume at $30^{\circ} \mathrm{C}$ for $60 \mathrm{~min}$.

Immunoprecipitation of lamin B1 was performed using protein A-conjugated Sepharose CL-4B beads (Pharmacia, Uppsala, Sweden) as described (19). Briefly, using a final volume of $600 \mu \mathrm{l}, 100 \mu \mathrm{l}$ of beads was incubated overnight at $4^{\circ} \mathrm{C}$ with $10 \mu \mathrm{l}$ of serum in NET 2 buffer. After incubation, beads were washed four times in NET 2, followed by overnight incubation with $10 \mu \mathrm{l}$ of translation product in $1 \mathrm{ml}$ NET 2. After five washes, bound proteins were eluted by boiling in Laemmli sample buffer under reducing conditions and analyzed by SDS-PAGE.

Statistical analyses. $\chi^{2}$ analyses were used to compare proportions. Due to the number of comparisons, only $P$ values $<0.01$ were considered significant. Sensitivity and specificity were calculated using all CFS patients $(n=60)$ and combining control groups I and II $(n=71)$.

\section{Results}

Demographic and clinical characteristics. The characteristics of the CFS patients and two control groups reported in this study are shown in Table I. These include features like duration of illness, concurrent psychiatric disorder, concurrent fibromyalgia and allergies, and positive Schirmer's test in the CFS group of patients. The age, gender, and race/ethnicity in the CFS group and control group I were comparable. Control group II was intended to represent a random sample of healthy individuals defined as regularly working people without disability.

Autoantibodies to nuclear and cytoplasmic antigens in serum of patients with CFS. IIF analysis of 60 sera from patients diagnosed with CFS showed that 52\% (31/60) contained antibodies against NE proteins by this technique (Table II). This involved a characteristic rim-like staining of the nuclear periphery in interphase cells and lack of labeling of mitotic chromosomes. The latter characteristic was used to exclude antibodies to dsDNA, which can also give peripheral nuclear labeling (25). We observed two types of nuclear rim labeling, examples of which are presented in Fig. 1: continuous or smooth nuclear rim staining which when superimposed over the domain of the nucleoplasm appears as a less intense fine grainy staining, typical of lamins and lamina-associated proteins (Fig. 1, $a-c$ ). The other type was a punctate nuclear rim 
Table II. Antibody Staining Patterns in CFS

\begin{tabular}{llll}
\hline & $\begin{array}{c}\text { CFS } \\
(n=60)\end{array}$ & $\begin{array}{c}\text { Control group I } \\
(n=30)\end{array}$ & $\begin{array}{c}\text { Control group II } \\
(n=41)\end{array}$ \\
\hline ANA positive*, $n(\%)^{\text {Nuclear rim staining*, } n(\%)}$ & $41(68)$ & $3(10)^{\ddagger}$ & $6(15)^{\ddagger}$ \\
$\begin{array}{l}\text { Nucleoplasmic staining*, } n(\%) \\
\text { Cytoplasmic staining, } \\
\quad \text { vimentin-like pattern*, } n(\%)\end{array}$ & $28(47)$ & $2(7)^{\S}$ & $1(2)^{\ddagger}$ \\
& & $4(13)^{\ddagger}$ & $1(2)^{\S}$ \\
\end{tabular}

${ }^{\ddagger}$ Designated group vs. CFS, $P<0.00001 .{ }^{\S}$ Designated group vs. CFS, $P<0.001$. "Designated group vs. CFS, $P<0.05$. *Titer of $1 / 50$ or greater.

staining associated with a coarser type of grainy staining, which is characteristic of the NPC proteins (Fig. 1, $d-f$ ).

In the sera positive for NE staining, 18 of 31 exhibited reactivity at a dilution of 1:50. Reactivity at a dilution of 1:100 was found in nine sera and four sera yielded antibody staining of $\mathrm{NE}$ at dilutions of 1:200 or greater.

In addition to the NE staining, 20 sera gave a thread-like, wavy cytoplasmic staining that seemed to abut the nuclear membrane and extend to the plasma membrane. This pattern is characteristic of members of the intermediate filament protein family. By double IIF, we colocalized the cytoplasmic staining pattern of these 20 CFS sera with vimentin (Table II). Although the labeling produced by antivimentin autoantibodies seemed to overlap with the staining of the NE, it possessed some unique features which were used to differentiate them (Fig. 2). Usually, the antivimentin antibodies labeled the nuclear rim incompletely (Fig. $2 b$ ), and with varying thicknesses, while anti-NE antibodies showed uniform labeling and uniform thickness (Fig. $2 a$ ). Moreover, the vimentin fibers aggregated in bundles around the nucleus, producing fluorescent labeling in many focal planes at the nuclear periphery, in contrast to the labeling of antigens restricted to the NE, which were observed in a more restricted focal plane at the nuclear rim. In our study, six of the CFS sera contained both the NE and vimentin type reactivities. All positive sera were exclusively of the $\mathrm{IgG}$ class and we were unable to detect any low titer IgM autoantibodies to either NE antigens or vimentin.

In addition to antibodies against the $\mathrm{NE}$ and vimentin, 28 of the 60 CFS sera were positive for staining of nucleoplasmic and nucleolar structures. These findings will be described in greater detail elsewhere. Examples are shown by CFS serum 411 (Fig. 3), which exhibited two nucleoplasmic staining patterns: one of these was a reticulated pattern, which covered the whole nucleus except for the nucleolus, and which titrated out at serum dilution of 1:800 (Fig. $3 d$ ); and another pattern with one to five bright dots per nucleus (Fig. $3 d$ ), and which had a titer $>1: 2,000$. The latter pattern colocalized with antibodies to $\mathrm{p} 80$ coilin (Fig. $3, h-j$ ). In addition, this specimen exhibited a cytoplasmic staining pattern, which did not colocalize with a mouse monoclonal antibody to vimentin (Fig. 3,e-g). The simultaneous presence of high-titered autoantibodies against nucleoplasmic or cytoplasmic cell components complicates the detection of anti-NE antibodies, and in such cases IIF alone is not sensitive enough to detect specific anti-NE antibodies.

In control group I consisting of 10 healthy individuals, 10 patients with major depression who did not meet the CFS case definition, and 10 patients with allergies, only one serum stained the nuclear rim weakly, while four sera (13\%) yielded low-titer antibodies to cytoskeletal components, which were colocalized with vimentin. In control group II consisting of 41
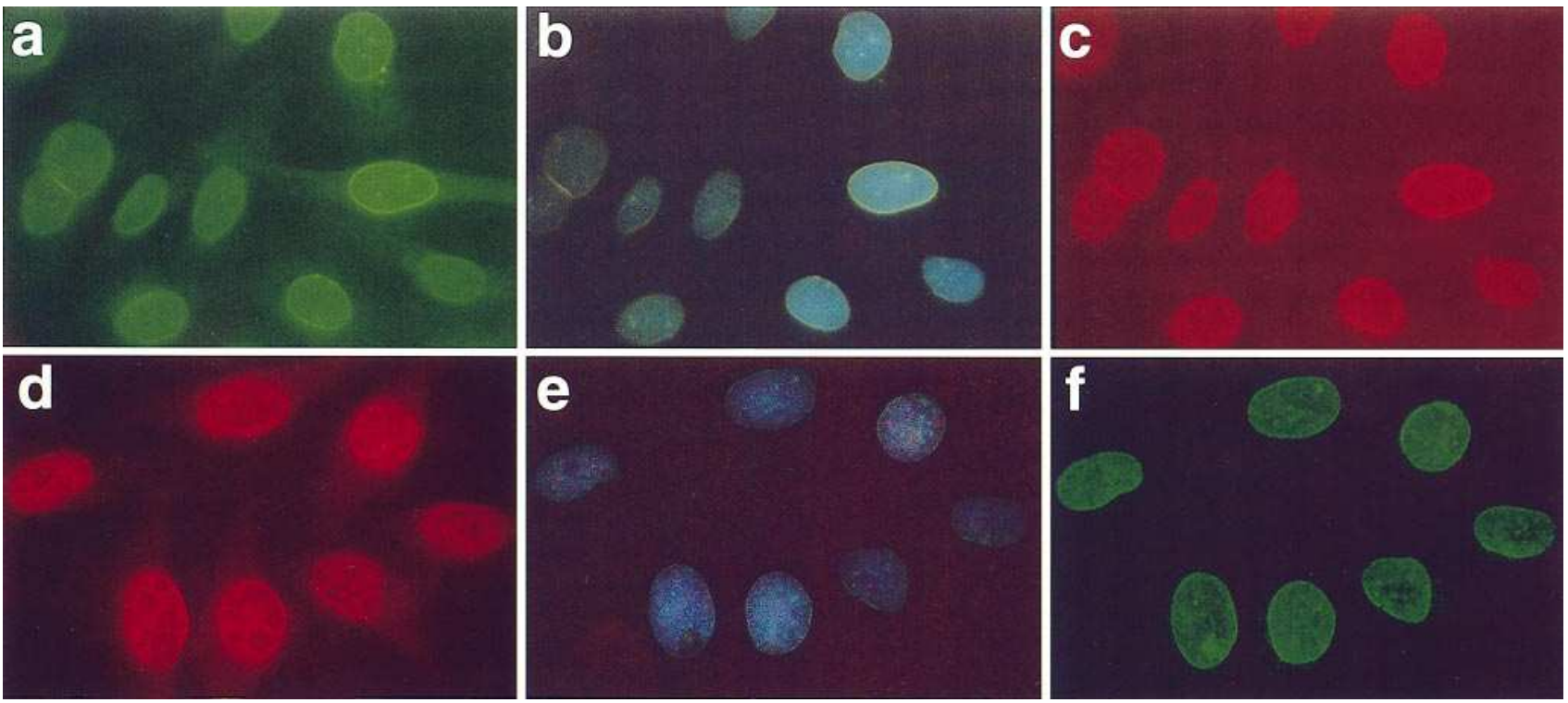

Figure 1. Representative anti-NE antibody patterns in immunofluorescence microscopy from CFS patients. Human HEp-2 cells were doublestained with CFS serum 555 (a) and hamster monoclonal antibody (RL29, IgG) to LAP 2 (c). Both antibodies show smooth and continuous, rimlike peripheral staining of the nucleus. In merged images $(b)$ yellow color denotes colocalization. When HEp- 2 cells were double stained with CFS serum $905(d)$ and mouse monoclonal antibody (RL1, IgM) to a group of NPC proteins $(f)$, both sera demonstrate punctate fluorescence at the nuclear rim with the merged image (e). The DNA-binding dye 4,6-diamidino-2 phenylindole (DAPI) (blue) was used to delineate the nucleoplasmic compartment. Merged images were performed with a triple filter. The human sera were diluted 1:100. Note that CFS serum $905(d)$ also shows nucleoplasmic staining, suggesting the presence of other antibodies. 

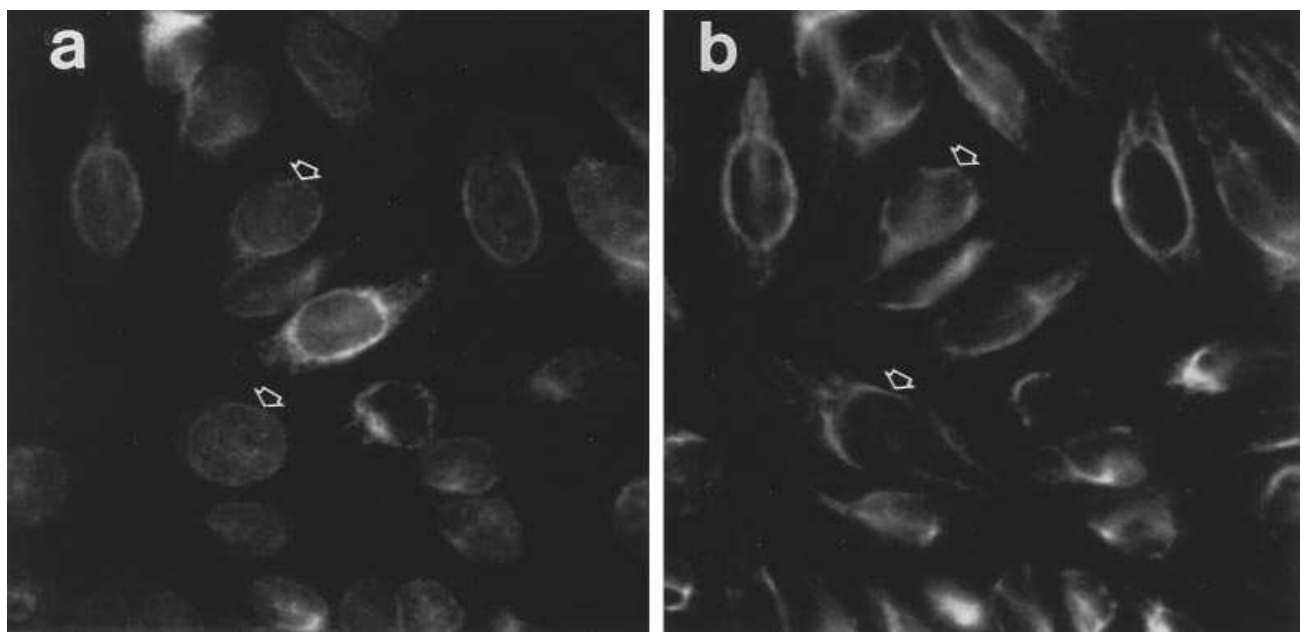

Figure 2. Double-IIF staining of human HEp-2 cells with CFS serum $441(a)$ and mouse monoclonal antibodies to vimentin (b). The NE-specific antibodies produced a labeling pattern characterized by a complete nuclear rim staining ( $a$, arrows). Antibodies to vimentin also stain the nuclear rim in some cells $(b)$ but the labeling of the nuclear rim is discontinuous in most cells ( $b$, arrows).

healthy blood donors, only one of the sera showed weak nuclear rim staining. Table II also shows the significance values between CFS and the control groups with regard to the different patterns of staining.

Immunoblotting. To establish the composition of antigens in the SWNE preparations and to determine the presence or absence of antigens that are frequently targets in other autoimmune diseases, we analyzed our SWNE preparations in immunoblotting against a battery of 10 reference human autoimmune sera which recognized 13 different autoantigens: Sm, U1
snRNP, SS-A/Ro, SS-B/La, rRNP, and Ku (Fig. 4, lanes 1-6), and also CENP-A, B, C, CENP-F, Jo-1, Scl-70, PCNA (not shown). The same sera were used to analyze MOLT-4 whole cell extracts in order to demonstrate that several nucleoplasmic autoantigens were absent in SWNEs. With the exception of a 37-kD band, recognized by a serum containing anti-rRNP antibodies (Fig. 4, lane 4), no reactivities with these reference autoimmune sera were observed with SWNEs. Since the outer nuclear membrane of the NE contains attached ribosomes and is continuous with the endoplasmic reticulum, it is likely that
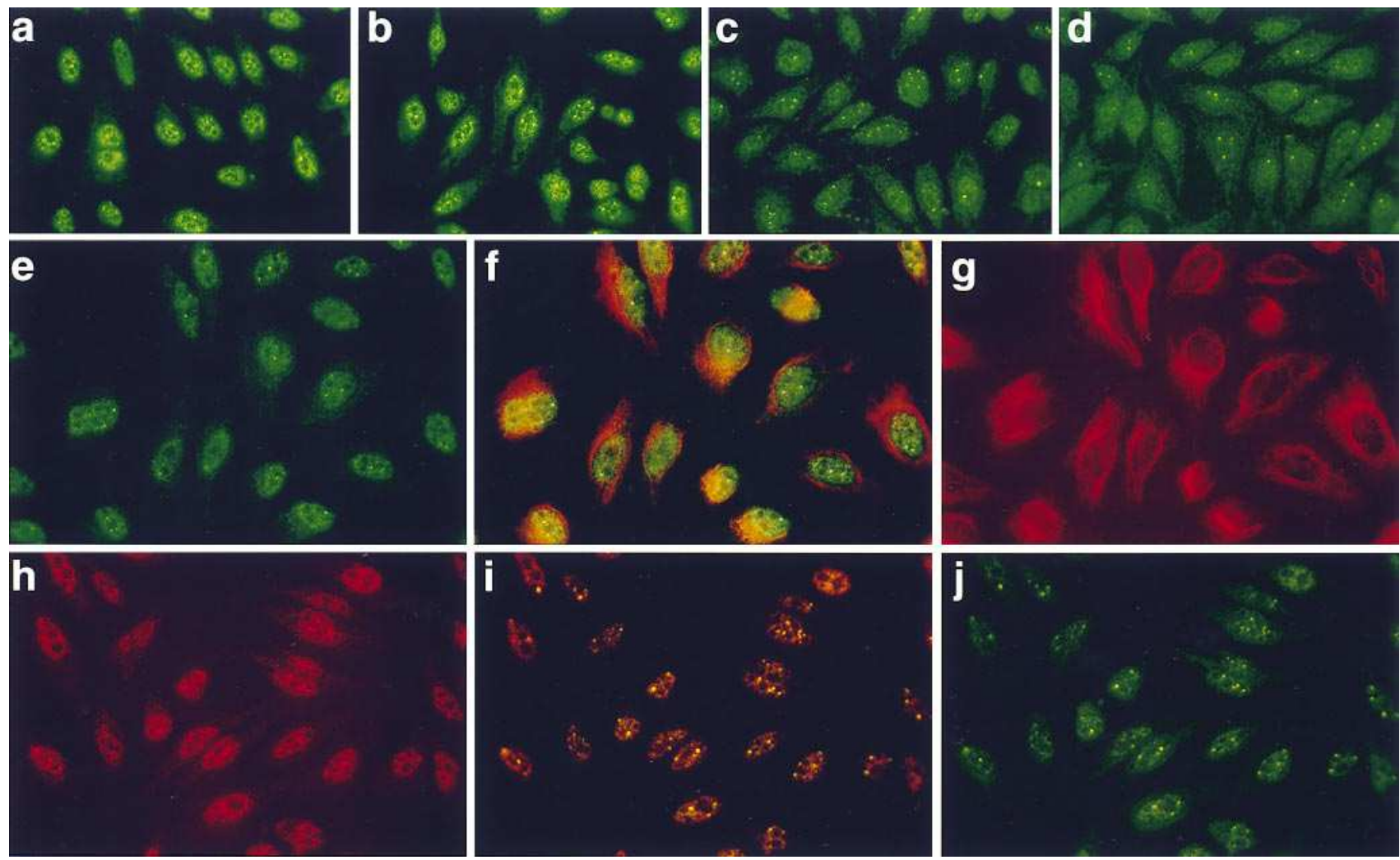

Figure 3. IIF staining of human HEp-2 cells with serial dilutions of CFS serum 411. The dilutions shown are: $a, 1: 50 ; b, 1: 100 ; c, 1: 400 ; d, 1: 800$. Double-staining of cells with CFS serum $(e)$ and with a mouse monoclonal antibody to vimentin $(g)$ does not show colocalization of the cytoplasmic patterns $(f$, merge). Double-staining of CFS serum $(h)$ with rabbit polyclonal antibody to $\mathrm{p} 80$ coilin $(j)$ shows colocalization of the nuclear dot pattern. In the merged image $(i)$, the yellow color denotes colocalization. 


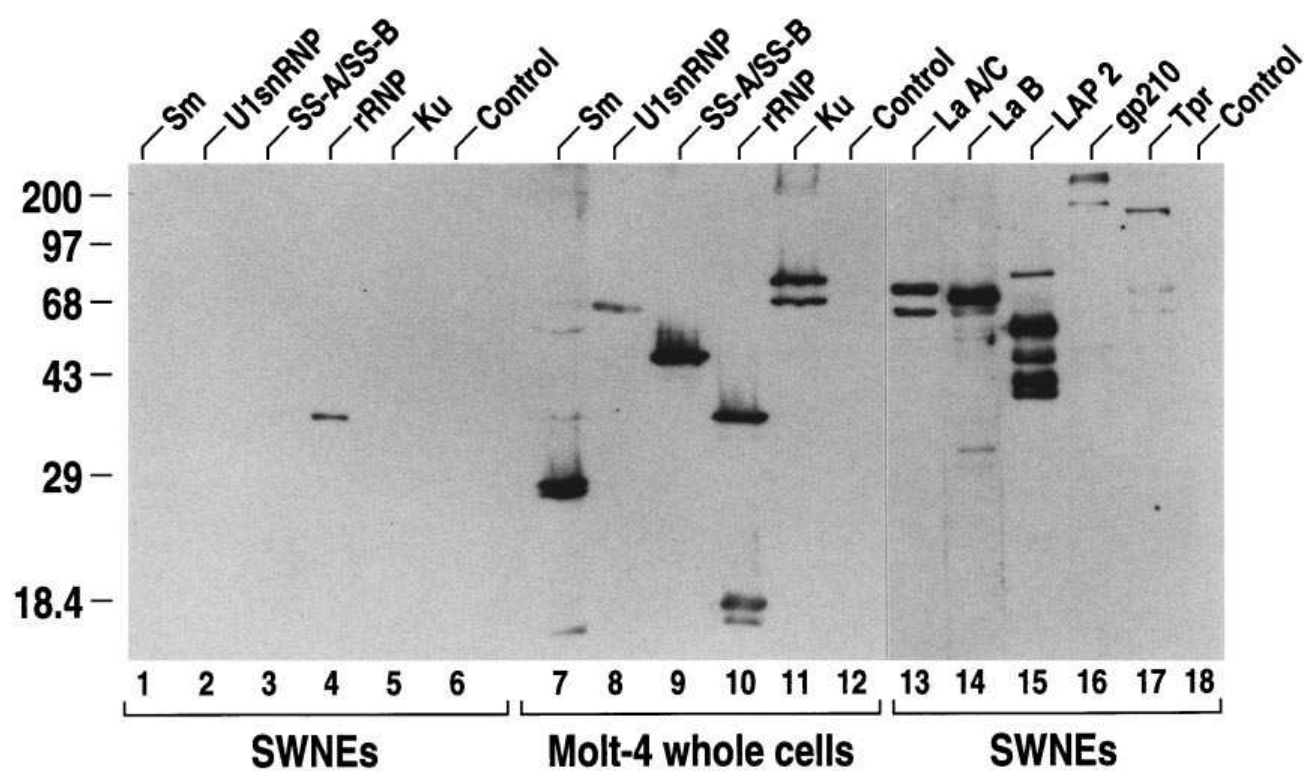

Figure 4. Characterization of antigens in rat liver SWNEs. Samples of SWNEs (lanes 1-6 and 13-18) or a total cell extract of MOLT-4 cells (lanes 7-12) was subjected to electrophoresis on the same SDS gel and processed for immunoblot analysis. Lanes were probed with 1:100 dilution of human sera with defined reactivity: anti-Sm, lanes 1 and 7; antiU1snRNP, lanes 2 and 8 ; antiSS-A/Ro and SS-B/La, lanes 3 and 9; antiribosomal (r) RNP, lanes 4 and 10 ; and anti-Ku, lanes 5 and 11. Control denotes lanes probed with normal human serum. Lanes 13-17, containing SWNEs, were probed with known reference sera containing autoantibodies to $\mathrm{NE}$ antigens: lamin $\mathrm{A} / \mathrm{C}$, lane 13 ; lamin B, lane 14; LAP 2, lane 15; gp210, lane 16; Tpr, lane 17. Molecular weight markers are designated on the left. the antiribosomal reactivity comes from the phosphoprotein component (PO) of the large ribosomal subunit, which exhibits this molecular size in SDS gel electrophoresis (for comparison see Fig. 4, lane 10). Two other ribosomal components, P1 and P2 which migrate as a doublet at $16 / 18 \mathrm{kD}$ (Fig. 4, see lane 10), were absent from the SWNE preparation. Thus, possibly with the exception of the PO component of ribosomal RNP, 12 of the common autoantigens which are targets of autoantibodies in specific autoimmune diseases were not detectable in the SWNE preparation. In contrast, lanes 13-18 of Fig. 4 demonstrate the presence of some peripheral and integral $\mathrm{NE}$ polypeptides in the SWNE preparation: lamins A and C (70and $60-\mathrm{kD}$ bands), lamin B (68-kD band), LAP 2 (a set of 80/ 53/43/41-kD bands), gp210 (210-kD band), and Tpr (translocated promoter region protein; major $175-\mathrm{kD}$ band and other minor degradation products). It is important to note that MOLT-4 whole cell extracts prepared in the standard way for detection of autoantibodies in sera of patients with systemic autoimmune diseases vary in the content of NE antigens so that the same sera used in Fig. 4 (lanes 13-17) would give highly variable results in immunoblotting of lamins or other NE proteins.

When sera from CFS patients were analyzed by immunoblotting against SWNEs, a number of different reaction patterns were observed, often involving two or more bands (Fig. 5). Since it was established that many known nuclear and cytoplasmic autoantigens were not detectable in the SWNE preparations, and in contrast, many integral and peripheral NE proteins were present, we characterized the reactive bands of CFS sera relative to the known mobilities of NE proteins in this system. $67 \%$ of the CFS patients were positive for NE reactivity, compared to $10 \%(5 / 51)$ found among the normal subjects in control groups I and II. In addition, none of the patients with chronic depression or atopy showed reactivity to NE proteins. The most frequently recognized band detected by the CFS sera migrated at $68 \mathrm{kD}$ (for example Fig. 5, lanes 7, 9, 10, 13, $18,22,23,26,29-32)$. This $68-\mathrm{kD}$ band migrated at a position between the two markers for lamins $\mathrm{A}$ and $\mathrm{C}$ in the control lanes (extreme right in each panel) and is typical for migration of lamin B (refer to Fig. 4, lanes 13 and 14). Blotting reactivity of sera varied in intensity. Three of the CFS sera recognized a 38-kD band, which comigrated with the PO component of the rRNP (Fig. 5, lanes 7, 14, 18). However, definitive confirmation that this $38-\mathrm{kD}$ band was the PO component of rRNP was not pursued.

Recognition of nuclear lamins by CFS antisera. Affinityeluted antibodies were obtained from the $68-\mathrm{kD}$ band, which comigrated with lamin B1. As shown in Fig. $6 \mathrm{~A}$, the antibodies that were isolated by immunoaffinity purification were found to rebind specifically to a $68-\mathrm{kD}$ component of SWNEs. They reacted with a single band, which comigrated with a band obtained with a control lamin B antiserum (Fig. $6 \mathrm{~A}$, lanes 2 and 3). Immunofluorescence staining of HEp-2 cells obtained with unfractionated patient serum is shown in Fig. $6 C$. The affinity-purified antibody reacted only with the NE. This experiment was reproduced three times with two different sera.

To confirm that the reactivities of CFS sera with the $68-\mathrm{kD}$ antigen were produced by anti-lamin B1 antibodies, we randomly selected sera with and without $68-\mathrm{kD}$ reactivity and analyzed them in immunoprecipitation with in vitro translated radiolabeled human lamin B1. The translated product revealed the expected electrophoretic mobility of lamin B1 (Fig. 7, lane T). Immunoprecipitation with defined anti-lamin B human serum as positive control was also performed (Fig. 7, lane 4). Seven CFS sera which were positive in immunoblotting with the $68-\mathrm{kD}$ band, three which were negative, and two normal human sera were tested. Five of the seven $68-\mathrm{kD}-$ positive CFS sera immunoprecipitated in vitro translated human lamin B1 (Fig. 7), while the rest of the sera were negative. These results confirmed that the NE reactivity of some CFS 
$123456789101112 r^{5} v^{p^{C}}$
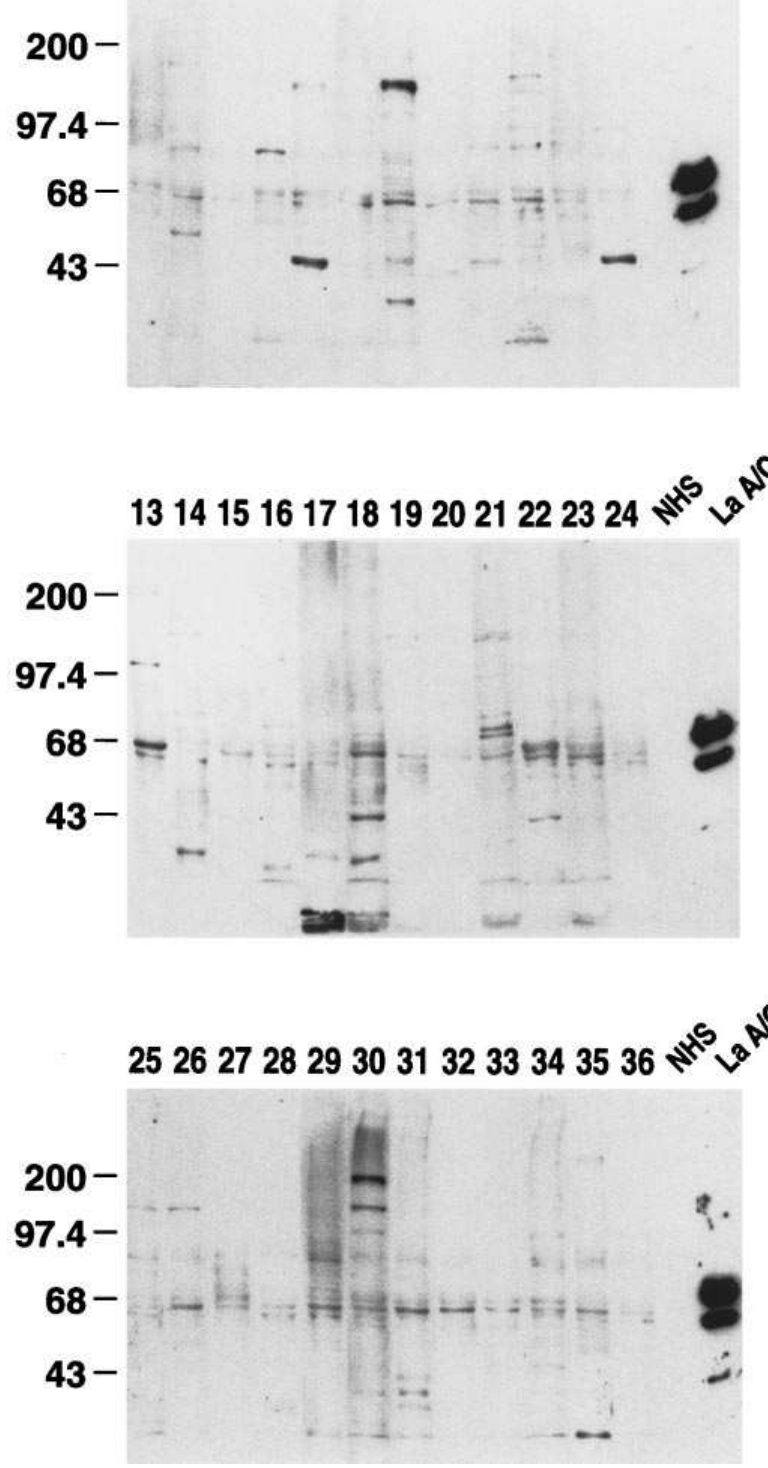

Figure 5. Detection of IgG autoantibodies against antigens of the NE in sera of a representable group of 36 patients with CFS by immunoblotting. Proteins of SWNEs were separated in a 7.5\% SDS-polyacrylamide gel and transferred to a nitrocellulose membrane. Blots were probed with a 1:100 dilution of patient sera (lanes 1-36), normal human sera $(N H S)$, or mouse monoclonal antibody (RL 12, IgM) to nuclear lamins A/C. Several other reference anti-NE sera were used as in Fig. 4, but are not shown in this micrograph. A common reaction was with a $68-\mathrm{kD}$ antigen, but reactive antigens of other molecular sizes were frequently present. Molecular weight markers are indicated on the left. Note that the $68-\mathrm{kD}$ band migrated between the two marker bands for lamins $\mathrm{A}$ and $\mathrm{C}$, in the control lanes (extreme right lane of each panel).

sera is against lamin B. The two CFS sera which were positive in Western blotting but negative in immunoprecipitation could have resulted from reactivity with a different NE antigen or with a lamin B epitope not revealed in the immunoprecipitation system, but this was not further analyzed.

\section{Discussion}

Of the 60 CFS patients examined in our study, about half had detectable levels of autoantibodies by immunofluorescence microscopy. The most frequently observed labeling was a perinuclear rim of enhanced staining intensity, a pattern suggestive of reactivity with NE antigens. Because HEp-2 cells in culture are monolayers and are flattened against the plane of the substratum, most focal planes that image the nucleus include the dorsal or ventral nuclear surface, as well as the nuclear edge. Hence, antigens that may be restricted to the NE give a picture of relatively uniform staining overlying the area of the nucleoplasmic domain but have a characteristic of accentuated peripheral rim labeling. As shown in Fig. 1, the rim-like peripheral nuclear labeling obtained with sera from CFS patients was very similar to the patterns obtained with experimentally produced monoclonal anti-NE antibodies.

The finding of characteristic NE staining in fluorescence immunohistology was confirmed by immunoblotting using isolated rat liver NEs. It should be noted that the immunoblotting studies would be detecting human autoantibodies cross-reacting with homologous rat antigens and recognizing epitopes conserved between human and rat species. There was a slightly higher incidence of antibodies to $\mathrm{NE}$ in immunoblotting $(67 \%)$ than in immunofluorescence $(52 \%)$ which could be related to the higher sensitivity of the former technique and also to the purification and concentration of $\mathrm{NE}$ antigens in the rat liver NE preparation. In a previous study (24) rat liver NE preparations were shown to provide a good source of reagents to identify novel autoimmune antigens of the NE. However, since the rat liver NE preparations contain more than NE proteins (such as the presumptive PO of rRNP), the conservative estimate of antibodies reactive with $\mathrm{NE}$ antigens might be closer to $52 \%$ than $67 \%$. The combination of the staining pattern and corresponding molecular weight estimation by immunoblotting provided the initial lead to the possibility that lamin B1 might be involved. This was confirmed by immunofluorescence results obtained with affinity-purified antibodies and immunoprecipitations with in vitro translated lamin B1. No antibodies to lamins A and C or to gp210, Tpr, and LAP 2 were detected in the CFS sera.

Contradictory results have been reported regarding the presence of ANA in CFS patients. The prevalence of ANAs in our study is higher than the previously reported data (9). Our CFS patients have a low level of ANA and anti-NE antibodies, some with titers in the 1:50 range. This is in contrast with reactivities observed in classic autoimmune diseases, such as lupus and Sjogren's syndrome, where the autoantibodies are usually high-titered and therefore readily detected by standard assays. For Western blotting, we used a highly purified antigen preparation (the SWNEs) which is far superior in detection of specific antibodies to NE components than unfractionated cell extracts, thus possibly explaining the higher frequency of autoantibodies among our samples. Among the components of the $\mathrm{NE}$, the known autoantigens include lamins $\mathrm{A} / \mathrm{C}$, lamin $\mathrm{B}$, gp210, Tpr, LAP 1 and 2, and p58/lamin B-binding protein (24-26). It is interesting that the NE-specific autoantibodies in CFS sera predominantly react with lamin B1.

Autoantibodies to NE proteins are relatively infrequent in routine ANA serology; their true prevalence is not known, and only a few of the antigens recognized by these antibodies have been characterized in detail. To date, a clinical association is 

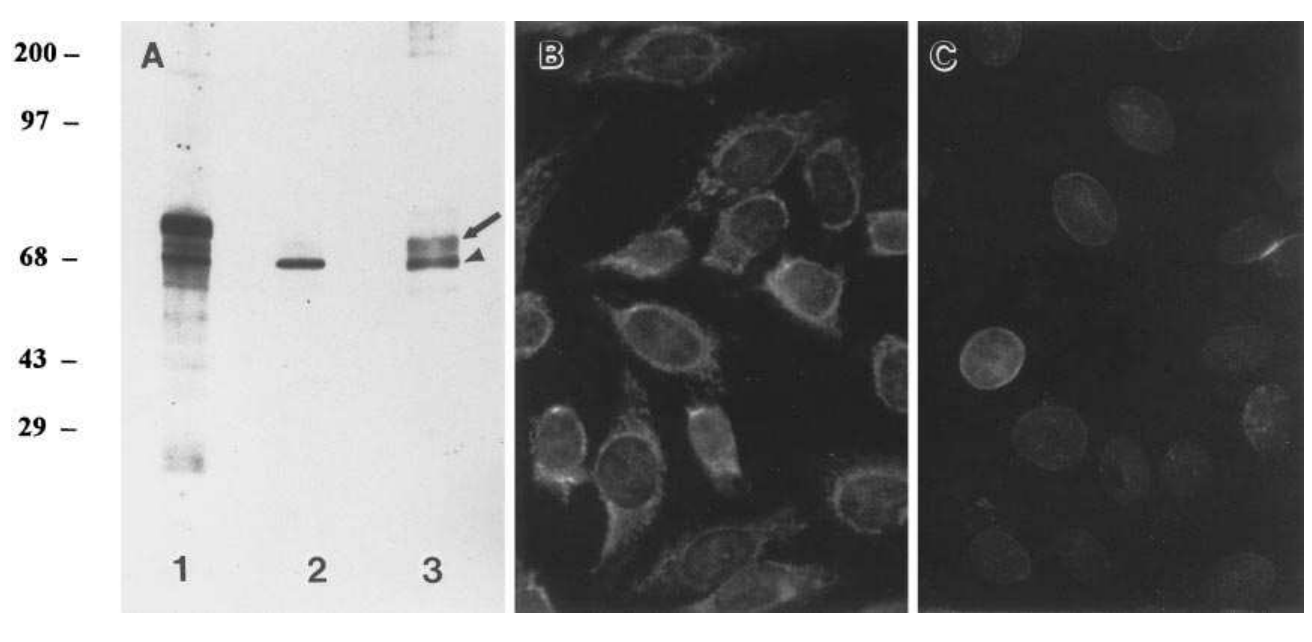

Figure 6. Analysis of affinitypurified antibodies reacting with a $68-\mathrm{kD}$ band of the NE fraction. Immunoblotting with unfractionated CFS serum 555 ( $A$, lane 1$)$, affinity-purified anti-68-kD antibody from CFS 555 ( $A$, lane 2$)$, and human antiserum with lamin $\mathrm{B} 1 / \mathrm{B} 2$ reactivity $(A$, lane 3$)$. Arrowhead indicates lamin B1, arrow indicates lamin B2. HEp-2 cells were used for IIF microscopy with unfractionated serum CFS $555(B)$ or the affinity-purified antibody $(C)$. The labeling in $C$ appears as a continuous fluorescent ring at the NE.

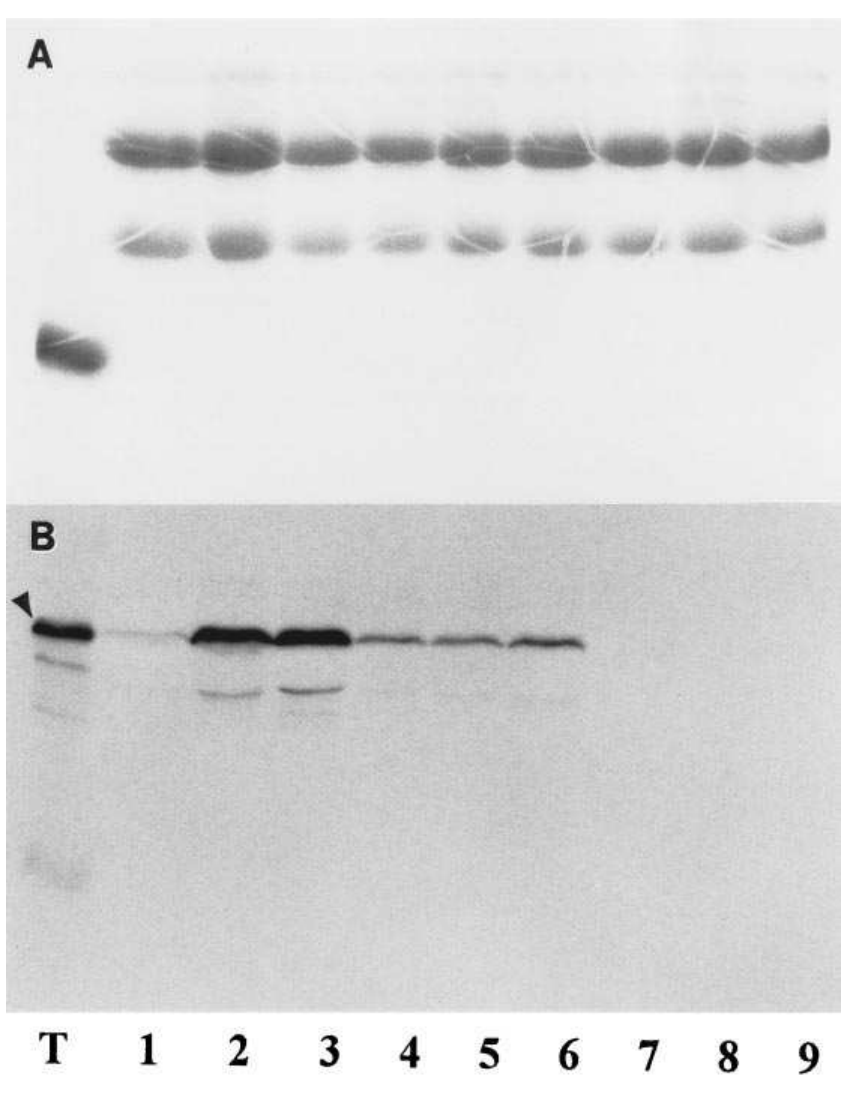

Figure 7. Immunoprecipitation of ${ }^{35} \mathrm{~S}$-labeled lamin B1, produced by in vitro transcription and translation. Immunoprecipitated ${ }^{35} \mathrm{~S}$-labeled lamin B1 was resolved on $12.5 \%$ polyacrylamide gel. $(A)$ Coomassie blue staining which identifies the $\operatorname{IgG}$ heavy and light chains (lanes 1-9) and an unidentified product in lane T. (B) Immunoprecipitation reactions obtained with five sera which were reactive with the $68-\mathrm{kD}$ band: 411 (lane 1), 532 (lane 2), 555 (lane 3), 658 (lane 5), 831 (lane 6); serum from an autoimmune disease patient with known anti-lamin B reactivity (lane 4 ); 2 CFS sera which were nonreactive with the 68-kD band (lanes 7 and 8 ), and a normal human serum (lane 9). $T$ corresponds to a lane loaded with the translation product. Full-length lamin B is indicated by an arrowhead. known only for autoantibodies to gp210 and p58, which appear in a subset of primary biliary cirrhosis patients $(27,28)$. Antibodies to nuclear lamins have been rarely characterized by examination of specific disease groups and have been previously reported in a small number of patients with diverse disease conditions. Most of these fall into the broad category of systemic rheumatic diseases $(29,30)$, chronic active hepatitis (31), and an unusual connective tissue disease subset characterized by steroid-responsive blood cytopenia, hepatitis, and brain or skin vasculitis (32). Studies in systemic lupus erythematosus patients showed that both the initiation and maintenance of lamin B antibody production are independent of the level of polyclonal B cell activation (33).

In this study we find that the NE in general, and lamin B1 in particular, become targets of the autoimmune response, raising the question of whether this cellular structure may be a target of the postulated ongoing immune system activation in CFS. Although such activation could be the result of various triggering agents, such as infections or environmental toxins, despite extensive investigations, infectious agents have not been definitely associated with CFS. However, some cases of CFS have been noted after L-tryptophan ingestion (34). It would be of interest to determine if CFS cases related to xenobiotic agents have similar immune reactivities with $\mathrm{NE}$ antigens as the general population of CFS patients. In this regard, Varga et al. (35) recently described a patient who developed autoantibodies to lamin $\mathrm{C}$ after L-tryptophan ingestion.

Future work should be directed at a better understanding of the autoimmune response of CFS patients to other NE antigens using recombinant NE proteins in Western blotting and ELISAs. Such studies examining larger series of patients could provide more definitive information on the utility of these autoantibodies in the diagnosis and classification of patients with CFS.

\section{Acknowledgments}

This is publication 9848-MEM from the Scripps Research Institute.

The assistance of J. Umali in the identification of the CFS patients is gratefully acknowledged. The Scripps Research Institute 
GCRC is acknowledged for samples of sera from healthy blood donors (NIH 7-MO1 RR00833).

This study was supported in part by grants from the National Institutes of Health and the National Institute of Mental Health. A. von Mikecz was the recipient of a fellowship from Deutsche Akademischer Austauschdienst (DAAD).

\section{References}

1. Holmes, G.P., J.E. Kaplan, M.M. Gantz, A.L. Komaroff, L.B. Schonberger, S.E. Straus, J.F. Jones, R.E. Dubois, C. Cunningham, J. Rundles, et al. 1988. Chronic fatigue syndrome: a working case definition. Ann. Intern. Med. 108:387-389.

2. Schluederberg, A., S.E. Straus, P. Petersen, S. Blumenthal, A.L. Komaroff, S.B. Spring, A. Landay, and D. Buchwald. 1992. Chronic fatigue syndrome research definition and medical outcome assessment. Ann. Intern. Med. 117: 325-333.

3. Fukuda, K., S.E. Straus, I. Hickie, M.C. Sharpe, J.G. Dobbins, A. Komaroff, and the International Chronic Fatigue Syndrome Study Group. 1994. The chronic fatigue syndrome: a comprehensive approach to its definition and study. Ann. Intern. Med. 121:953-959.

4. Buchwald, D., P. Umali, J. Umali, P. Kirth, T. Pearlman, and A.L. Komaroff. 1995. Chronic fatigue and the chronic fatigue syndrome: prevalence in a $\mathrm{Pa}$ cific Northwest health care system. Ann. Intern. Med. 123:81-88.

5. Subira, M.L., A. Castilla, M.-P. Civeira, and J. Prieto. 1989. Deficient display of CD3 on lymphocytes of patients with chronic fatigue syndrome. J. Infect. Dis. 160:165-166.

6. Klimas, N.G., F.R. Salvato, R. Morgan, and M.A. Fletcher. 1990. Immunologic abnormalities in chronic fatigue syndrome. J. Clin. Microbiol. 28:14031410 .

7. Straus, S.E., J.K. Dale, J.B. Peter, and C.A. Dinarello. 1989. Circulating lymphocyte levels in chronic fatigue syndrome. J. Infect. Dis. 160:1085-1086.

8. Landay, A.L., C. Jessop, E.T. Lennette, and J.A. Levy. 1991. Chronic fatigue syndrome: clinical condition associated with immune activation. Lancet. 338:707-712.

9. Bates, D.W., D. Buchwald, J. Lee, P. Kith, T. Doolittle, C. Rutherford, W. Hallowell, P.H. Schur, M. Wener, D. Wybenga, et al. 1995. Clinical laboratory test findings in patients with chronic fatigue syndrome. Arch. Intern. Med. 155:97-103.

10. Jones, J.F. C.G. Ray, L.L. Minnich, M.J. Hicks, R. Kibler, and D.O. Lucas. 1985. Evidence for active Epstein-Barr virus infection in patients with persistent, unexplained illnesses: elevated anti-early antigen antibodies. Ann. Intern. Med. 102:1-7.

11. Straus, S.E., G. Tosato, G. Armstrong, T. Lawley, D.T. Preble, W. Henle, R. Davey, G. Pearson, J. Epstein, I. Brus, and M. Blaese. 1985. Persisting illness and fatigue in adults with evidence of Epstein-Barr virus infection. Ann. Intern. Med. 102:7-16.

12. Calabrese, L.H., M.E. Davis, and W.S. Wilke. 1994. Chronic fatigue syndrome and a disorder resembling Sjogren's syndrome: preliminary report. Clin. Infect. Dis. 18(Suppl. 1):S28-S31.

13. Robins, L.N., and J.E. Helger. 1985. Diagnostic Interview Schedule (DIS): version III-A. St. Louis, Department of Psychiatry, Washington University School of Medicine.

14. American Psychiatric Association Diagnostic and Statistical Manual of Mental Disorders, 3rd ed. (revised). Washington, D.C., American Psychiatric Association, 1987

15. Senior, A., and L. Gerace. 1988. Integral membrane proteins specific to the inner nuclear membrane and associated with the nuclear lamina. J. Cell Biol. 107:2029-2036.
16. Foisner, R., and L. Gerace. 1993. Integral membrane proteins of the nuclear envelope interaction with lamins and chromosomes and binding is modulated by mitotic phosphorylation. Cell. 73:1267-1279.

17. Snow, C.M., A. Senior, and L. Gerace. 1987. Monoclonal antibodies identify a group of nuclear pore complex glycoproteins. J. Cell Biol. 104:11431156.

18. Andrade, L.E.C., E.M. Tan, and E.K.L. Chan. 1993. Immunocytochemical analysis of the coiled body in the cell cycle and during cell proliferation. Proc. Natl. Acad. Sci. USA. 90:1947-1951.

19. Pollard, K.M., E.C.L. Chan, B.J. Grant, K.F. Sullivan, E.M. Tan, and C.A. Glass. 1990. In vitro posttranslational modification of lamin B cloned from a human T-cell line. Mol. Cell. Biol. 10:2164-2175.

20. Gerace, L., Y. Ottaviano, and C. Kondor-Koch. 1982. Identification of a major polypeptide of the nuclear pore complex. J. Cell Biol. 95:826-837.

21. Imai, H., K. Furuta, G. Landberg, K. Kiyosawa, L.F. Liu, and E.M. Tan. 1995. Autoantibody to DNA topoisomerase II in primary liver cancer. Clin. Cancer Res. 1:417-424.

22. Laemmli, U.K. 1970. Cleavage of structural proteins during assembly of the head of bacteriophage $\mathrm{T}_{4}$. Nature (Lond.). 227:680-685.

23. Senecal, J.-L., and Y. Raymond. 1992. Autoantibodies to major and minor nuclear lamins are not restricted to autoimmune diseases. Clin. Immunol. Immunopathol. 63:115-125.

24. Konstantinov, K.N., R. Foisner, D. Byrd, F.-T. Liu, W.-M. Tsai, A Wiik, and L. Gerace. 1995. Integral membrane proteins associated with the nuclear lamina are novel autoimmune antigens of the nuclear envelope. Clin. Immunol. Immunopathol. 74:89-99.

25. Tan, E.M. 1989. Antinuclear antibodies: diagnostic markers for autoimmune diseases and probes for cell biology. Adv. Immunol. 144:93-151.

26. Worman, H.J., and J.-C. Courvalin. 1991. Autoantibodies against nuclear envelope proteins in liver disease. Hepatology. 14:1269-1279.

27. Courvalin, J.-C., K. Lassoued, H. Worman, and G. Blobel. 1990. Identification and characterization of autoantibodies against the nuclear envelope lamin B receptor from patients with primary biliary cirrhosis. J. Exp. Med. 172: 961-967.

28. Courvalin, J.-C., K. Lassoued, E. Bartnik, G. Blobel, and R.W. Wozniak. 1990. The $210-\mathrm{kD}$ nuclear envelope polypeptide recognized by human autoantibodies in primary biliary cirrhosis is the major glycoprotein of the nuclear pore. J. Clin. Invest. 86:279-285.

29. Reeves, W.H., N. Chaudhary, A. Salerno, and G. Blobel. 1987. Lamin B autoantibodies in sera of certain patients with systemic lupus erythematosus. $J$. Exp. Med. 165:750-762.

30. Konstantinov, K., P. Halberg, A. Wiik, M. Hoier-Madsen, P. Wantzin, S. Ullman, and Z. Galcheva-Gargova. 1992. Clinical manifestations in patients with autoantibodies specific for nuclear lamin proteins. Clin. Immunol. Immunopathol. 62:112-118.

31. Wesierska-Gadek, J., E. Penner, E. Hitchman, and G. Sauerman. 1988 Antibodies to nuclear lamins in autoimmune liver disease. Clin. Immunol. Immunopathol. 19:107-115.

32. Lassoued, K., M.-N. Guilly, F. Danon, C. Andre, D. Dhumeaux, J-P. Clauvel, J.-C. Brouet, M. Seligman, and J.-C. Courvalin. 1988. Antinuclear antibodies specific for lamins: characterization and clinical significance. Ann. Intern. Med. 108:829-833.

33. Chou, C.-H., S.A. Ali, R. Roubey, J. Buyon, and W.H. Reeves. 1991. Onset and regulation of anti-lamin B autoantibody production is independent of the level of polyclonal activation. Autoimmunity. 8:297-305.

34. Valesini, G., R. Priori, F. Conti, F.L. Luan, C. Ardino, and F. Balsano. 1994. Chronic fatigue syndrome: a peculiar evolution of eosinophilia myalgia syndrome following treatment with L-tryptophan in four Italian adolescents. Eur. J. Pediatr. 153:344-346.

35. Varga, J., G.G. Maul, and S.A. Jimenez. 1992. Autoantibodies to nuclear lamin $\mathrm{C}$ in the eosinophilia-myalgia syndrome associated with L-tryptophan ingestion. Arthritis Rheum. 35:106-109. 